\title{
Eclosión de huevos de artemia (Artemia franciscana Kellogg) a nivel de laboratorio en la Universidad Nacional Agraria, Managua, Nicaragua
}

\section{Hatching of brine shrimp eggs (Artemia franciscana Kellogg) in the laboratory of Universidad Nacional Agraria, Managua, Nicaragua}

\author{
Ing. Francisco Reyes Flores ${ }^{1}$, Lic. Rosa María Reyes ${ }^{1}$, Lic. Heraldo Salgado ${ }^{2}$, Harvin Cornejo ${ }^{3}$ \\ ${ }^{1}$ Docentes Investigadores FARENA/UNA \\ ${ }^{2}$ Técnico Investigador del Laboratorio de Ciencias Biologicas FARENA/UNA \\ ${ }^{3}$ Ingeniero Forestal
}

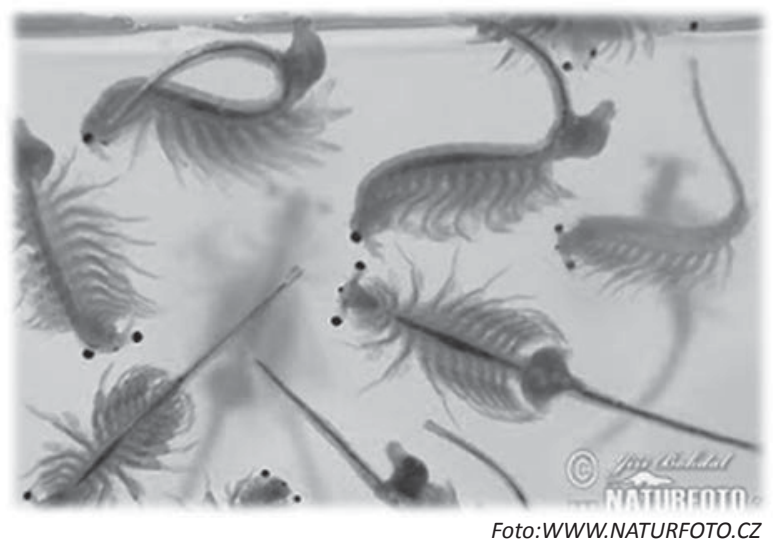

\section{RESUMEN}

En el estudio de eclosión de Artemia franciscana a nivel de laboratorio, tuvo como objetivo determinar el tipo de tratamiento que se adapta a condiciones de laboratorio brindando buenos resultados para la eclosión del crustáceo Artemia (Artemia franciscana Kellogg). La metodología utilizada consistió en la toma de datos en el laboratorio evaluando los siguientes tratamientos: Toma de pH; Toma de temperatura; Salinidad y Medición de Sobrevivencia. Una vez establecido el ensayo se procedió a colocar los huevo de Artemia, dejándolos por un periodo de 48 horas para iniciar el proceso eclosivo del crustáceo. El manejo que se aplicó en el estudio consistió en tomar datos de temperatura y del $\mathrm{pH}$ para llevar un control y seguimiento a la eclosión de Artemia; así como el control de la alimentación a base de una dieta de pastilla de alga Spirulina máxima triturada. De acuerdo al análisis estadístico realizado con metodología de DUNCAN, correlación de Pearson y procesado en el programa estadístico INFOSTAT indican que la mejor eclosión se registró en el tratamiento D con un $65.40 \%$ dando pautas de ser el tratamiento que mejor se adaptó a las condiciones de laboratorio. La sobrevivencia de los otros tratamientos es positiva y adaptable a condiciones de laboratorio donde es recomendable utilizar recipientes con mayor capacidad de almacenamiento de agua. Recomendándose para futuros cultivos de Artemia llevar a cabo el manejo ya implementado en el Laboratorio de Ciencias Biológicas de la Universidad Nacional Agraria para un mayor rendimiento en la sobrevivencia del estudio y seguir proporcionando información para la eclosión de dicho crustáceo que representa una opción alimenticia de bajo impacto a productores pesqueros del país.

Palabras clave: crustáceo, alga, laboratorio, sobrevivencia, ciencias biológicas.

Recibido: 7 de abril del 2016

Aceptado: 18 de agosto del 2016

\begin{abstract}
In the study of hatching of Artemia franciscana at laboratory level, aimed to determine the type of treatment that fits laboratory conditions giving good results for hatching the brine shrimp, Artemia (Artemia franciscana Kellogg). The methodology consisted of data collection in the laboratory evaluated by the following treatments: Taking pH; Taking temperature; Salinity; Survivorship measurement. Once established the study proceeded to put the egg of Artemia, which began to hatch iu 48 hours to start the process eclosivo crustacean. Management that was applied in the study consisted of taking data of temperature and $\mathrm{pH}$ to track and monitor the hatching of Artemia and control feed consisting base diet pill alga Spirulina maxima crushed. According to statistical analysis using methodology DUNCAN, Pearson correlation and processed in the INFOSTAT statistical program indicate that the best hatching was recorded in treatment $\mathrm{D}$ with $65.40 \%$ giving guidelines to be the treatment best adapted to the conditions of laboratory. The survival of other treatments is positive and adaptable to laboratory conditions where it is advisable to use containers with larger capacity water storage. It is recommended for future crops Artemia can carry out the management and implemented in the Laboratory of Biological Sciences of the National Agricultural University for higher performance in the survival of the study, and continue to provide information to the emergence of this crustacean food option for the low impact fishing producers in the country.
\end{abstract}


$\mathrm{E}$ l crustáceo artemia (Artemia franciscana Kellogg) es originario del norte de América hasta Centro América y el Caribe. Este crustáceo es alimento natural de especies de origen marino debido a que su hábitat natural es el mar y los lagos de concentración salina que se encuentran en Norte América, las propiedades que posee $A$. franciscana como alimento vivo de peces, es proporcionar al pez que lo consuma, una dieta rica en proteínas y vitaminas que mejoraran la alimentación del pez y su ciclo reproductivo (Castro, 2005).

A nivel mundial $A$. franciscana se ha reproducido en Estados Unidos, Centro América y el Caribe así como en ciertos países de Europa y Asia que comercializan A. franciscana como alimento vivo para acuarios, criaderos y santuarios de peces (Castro, 2005). Es importante destacar que Artemia también es alimento de toda la cadena trófica de zooplancton acuático.

En Nicaragua en el año 1989 la Organización de las Naciones Unidades para la Alimentación y la Agricultura (FAO) realizó dos estudios que informan de la reproducción artesanal de A. franciscana discutiendo generalidades de esta. Este crustáceo es una alternativa de alimento para peces, que vendría hacer una opción de alimentación que ayudaría a la reproducción y producción de peces mejorando su crianza debido a que se les daría un alimento vivo y no tratado industrialmente, lo que además brindaría un acuario más limpio para el pez que lo ingiriera (APROMAR, 2004). En el Laboratorio de Ciencias Biológicas de la Universidad Nacional Agraria (UNA) se logró eclosionar el crustáceo A. franciscana para contribuir a la generación de información científica debido a que existe poca información de este crustáceo en nuestro país.

\section{MATERIALES Y MÉTODOS}

El presente estudio se llevó a cabo en el laboratorio de Ciencias Biológicas de la Universidad Nacional Agraria, perteneciente a la Facultad de Recursos Naturales y del Ambiente (FARENA).

El estudio consiste en un Diseño Completamente Aleatorio trifactorial con una réplica por tratamiento (DCA), se asume que las unidades experimentales de una población se asignan al azar a grupos que generalmente son llamados tratamientos (Universidad de Colombia, 2002), en nuestro caso los tratamientos son la toma de datos del ensayo en estudio (figura 1).

Tratamiento B Tratamiento C Tratamiento D

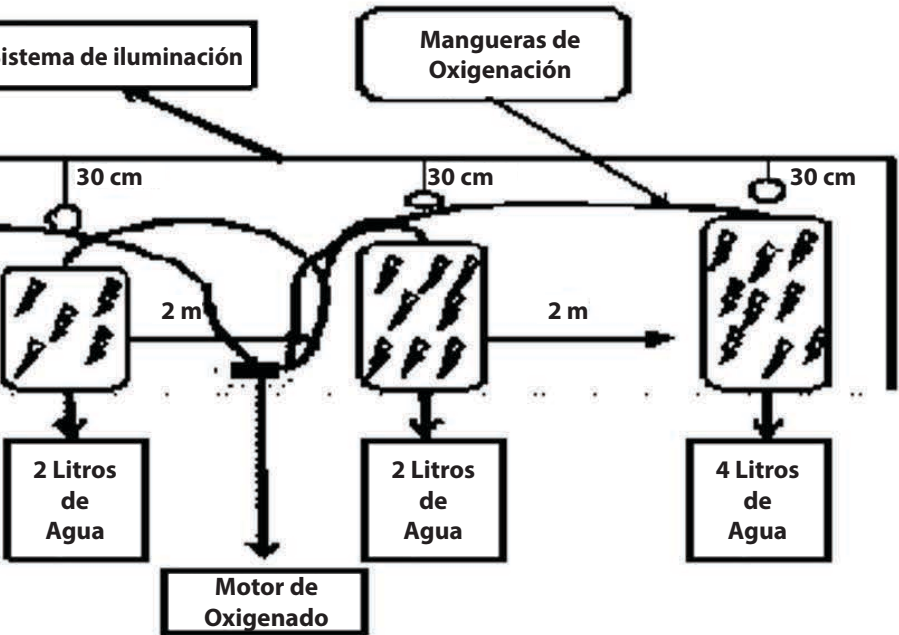

Oxigenado

Figura 1. Esquema de establecimiento del ensayo de Artemia franciscana en el Laboratorio de Ciencias Biológicas de la Universidad Nacional Agraria, 2015.

Para el manejo para la eclosión de huevos de artemia a nivel de laboratorio, se llevó a cabo en tres etapas:

Etapa 1, establecimiento. En primera instancia se procedió a desinfectar los cuatro frascos utilizados para los tratamientos lavándose con agua fría y posteriormente se pusieron a hervir en agua caliente por 3 minutos cada uno. Paralelo a esta actividad se colocó agua tomada de la llave en un recipiente a descolorar por 24 horas.

Una vez los frascos fueron desinfectados se colocaron a una distancia de $2 \mathrm{~m}$, entre ellos, con cantidades de agua que iban de un litro a cuatro litros, dejándolos en el sitio donde se ubicarían durante el periodo de ensayo, se colocó un marco de madera con un circuito eléctrico complejo y sencillo de hacer, para colocar tres bujías de 25 watts que se ubicarían en tres de los cuatro tratamientos en estudio.

Se colocó un motor HP 200 para producir oxigeno que se distribuyó por una red de mangueras instaladas desde el motor hasta los frascos para propiciar la producción de oxígeno en el agua. Se procedió a colocar 10 gramos de sal pura sin yodo, para controlar la acides del agua, el aire bombeado por el motor permitió acelerar la disolución de los granos de sal.

Etapa 2, monitoreo. El monitoreo se realizaba desde las 8:00 am hasta las 5:00 pm diario durante la duración del estudio, el objetivo de este proceso se centraba en observar, que el funcionamiento del motor fuera correcto, las mangueras estuviesen limpias, que el nivel de agua de los frascos se mantuviera a niveles establecidos previamente en la etapa 1 , los cuales se ven disminuidos por evaporación y al momento de realizar la limpieza de los frascos. 
La limpieza de los frascos se realizaba semanalmente para evitar acumulación de residuos alimenticios en el frasco, puesto que esto podría llegar a afectar al crustáceo en observación.

A las 10 de la mañana y 4 de la tarde, se procedía a la toma de datos, esto para mantener un registro de la temperatura y el $\mathrm{pH}$, con el objetivo de tener una media de temperatura y pH a la que se dio la eclosión de los huevos de Artemia franciscana.

Etapa 3, recolección de datos. En esta etapa es donde se hacen conteos de la cantidad de huevos de Artemia eclosionados y la cantidad de huevos no eclosionados, en esta etapa se realizaban los cálculos de sobrevivencia por tratamiento. Durante los 30 días que duro el ensayo, diariamente se tomaba la temperatura y $\mathrm{pH}$ registrándolos en formatos establecidos para luego ser procesados en programas estadísticos.

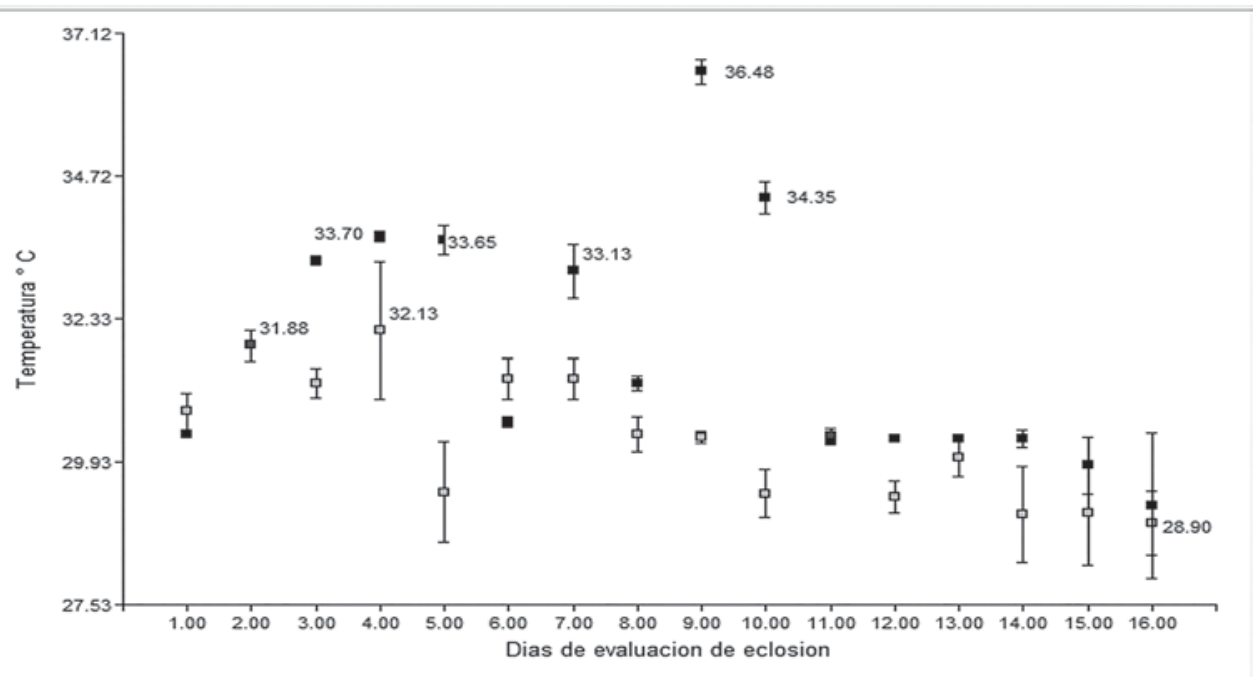

Figura 2. Variación de temperatura en los días de estudio del ensayo. Para procesar los datos se utilizó el INFOSTAT, y programa Excel, utilizando metodologías de Duncan y correlación de Pearson.

\section{RESULTADOS}

Manejo para tratamiento en eclosión. De los cuatro tratamientos colocados a eclosionar el tratamiento que dio mejores resultados con respecto a los otros fue el tratamiento D. Este tratamiento se encontraba en un recipiente con capacidad de almacenamiento de agua de 4 litros, lo que permitió se diera un mayor número de eclosión, al existir mayor número de huevos eclosionados con un 65.4 \% lo que ha dejado en evidencia que a mayor tamaño del recipiente mayor número de eclosiones.

A este tratamiento se le dio un manejo regulado que consistió en retirar el residuo de alimento que se almacenaba al fondo del recipiente con ayuda de una jeringa para evitar causar un posible estrés al crustáceo en eclosión.

Análisis de temperatura de los tratamientos a las 10:00 am y 4:00 pm. Los valores de temperatura diaria para el tiempo de evaluación de la eclosión muestran diferencias entre días y el momento de registro de esta variable. La temperatura por la tarde muestra los valores más altos con un rango entre 29.02 ${ }^{\circ} \mathrm{C}$ y $36.48{ }^{\circ} \mathrm{C}$; con $29.02{ }^{\circ} \mathrm{C}$ como valor más bajo obtenido el día 16 , y $36.48{ }^{\circ} \mathrm{C}$ como valor más alto registrado el día 9; aun así los valores de temperatura por la tarde muestran baja variabilidad en la muestra (figura 1) 
Análisis de pH de los tratamientos a las 10:00 am y 4:00 pm. La variable pH registrada en horas de la mañana, muestra diferencias altamente significativas entre los días de evaluación; distribuyéndose en un rango entre 7.70 y 8.32 punto de acides, lo que indica que los sustratos de eclosión de Artemia estuvieron ligeramente alcalino hasta medianamente alcalino.

El valor medio del $\mathrm{pH}$ para todo el periodo de evaluación fue de 8.05 punto de acides, presentando alta variabilidad entre días. Para el caso de los valores de $\mathrm{pH}$ tomados por la tarde, se obtuvo un valor medio de 9.19 punto de acides, para todo el periodo de eclosión, y por presentar alta igualdad en los valores diarios, no se encontró diferencias significativas (figura 4)

Porcentaje de sobrevivencia de Artemia a los 16 días. El porcentaje de sobrevivencia según los tratamientos se tomó a los 16 días de ser establecido el ensayo haciendo graficos de sobrevivencia (figuras 6 y 7). Aplicando las siguientes formulas se obtiene el porcentaje de sobrevivencia. \% mortalidad: Número de individuos / total de individuos * 100. Para obtener la sobrevivencia se utiliza la siguiente formula: \% sobrevivencia: $N$ - \% de mortalidad

1) El tratamiento A, a los 16 días obtuvo una sobrevivencia del 54.0.7\% tomando en cuenta que se pusieron 1500 huevos a eclosionar y eclosionaron un total de 811 individuos y no eclosionaron 689 huevos.

2) El tratamiento B a los 16 días obtuvo una sobrevivencia de $58.2 \%$ colocán-

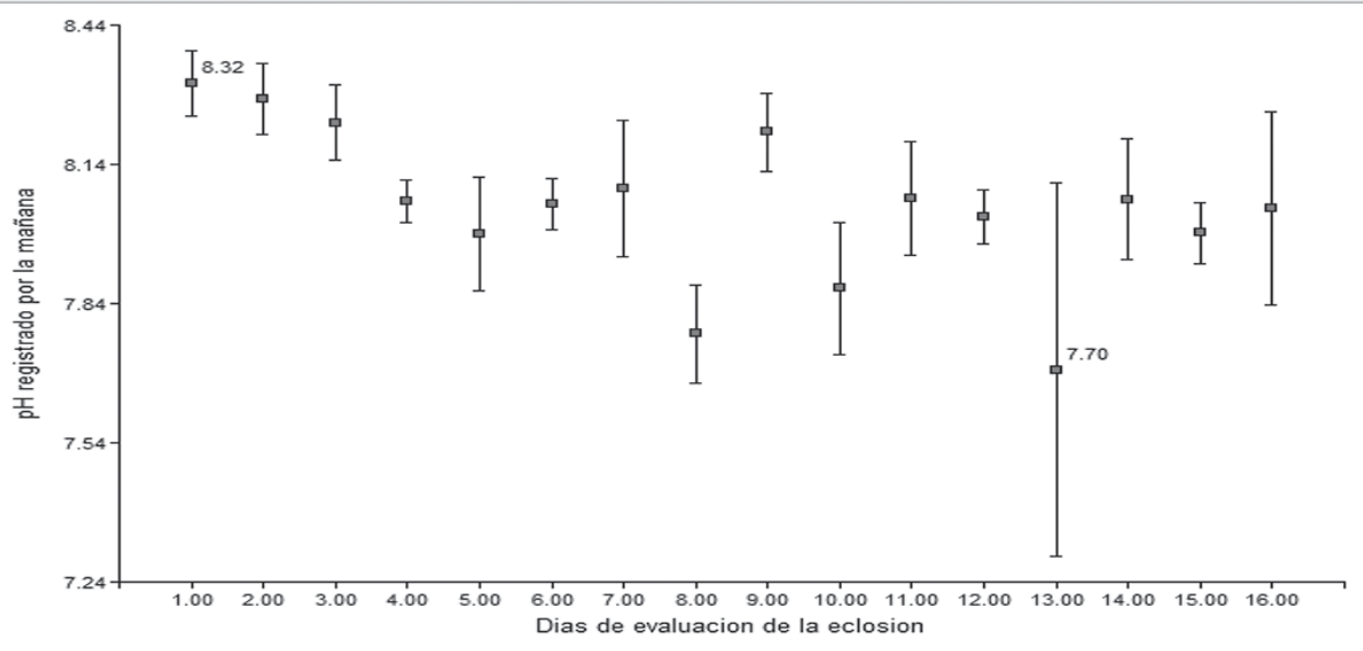

Figura 4. Variacion de $\mathrm{pH}$ en los dias de estudio de Artemia durante evaluacion del ensayo.

En la figura 5, se puede observar la asociación de la toma de $\mathrm{pH}$ por la mañana y de la tarde haciendo una asociación a los tratamientos del ensayo para determinar la significancia de esta variable en el ensayo. dose 1500 huevos de Artemia a eclosionar donde hubo una eclosión de 873 huevos y una no eclosión de 627 huevos.

3) El tratamiento $\mathrm{C}$ a los 16 días obtuvo una sobrevivencia de 58.60\% colocándose a eclosionar 1500 huevos de Artemia eclosionando 879 huevos y no eclosionando 621. 4) El tratamiento $D$ a los 16 días obtuvo una sobrevivencia de 65.40\% colocándose a eclosionar 1500 huevos de Artemia eclosionando un total de 981 y no eclosionando 519 huevos.

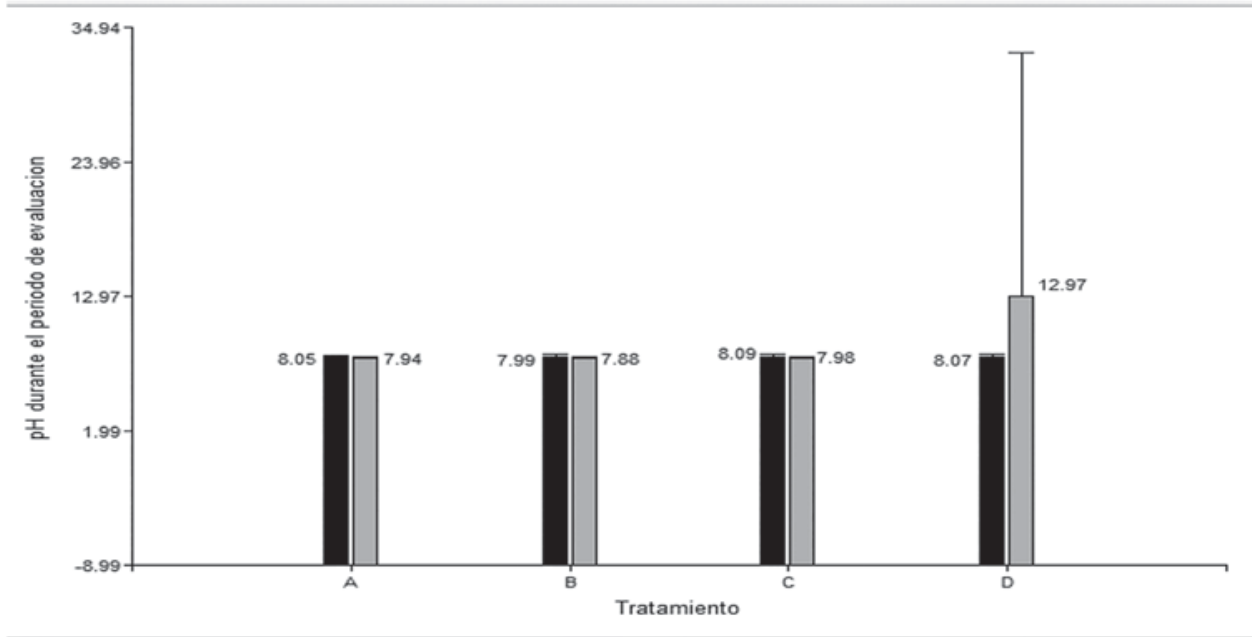

Figura 5. Asociacion de $\mathrm{pH}$ con los tratamientos, para determinar significancia en el ensayo. 


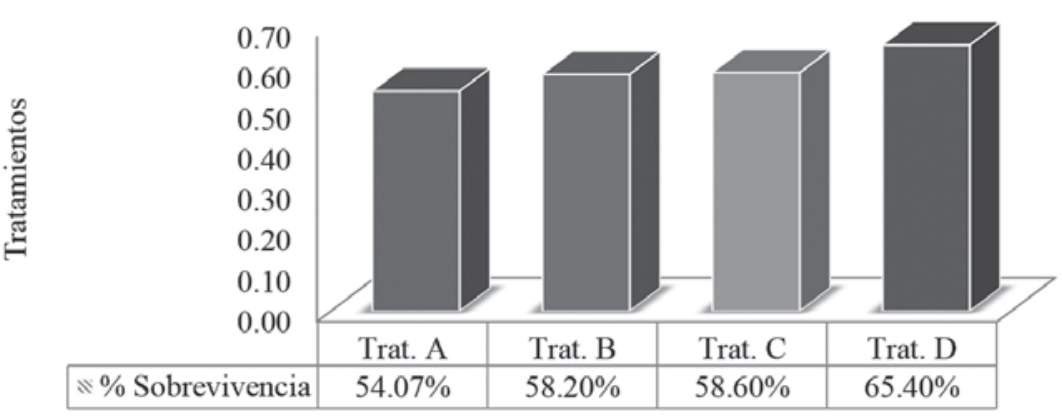

Figura 6. Porcentaje de sobrevivencia de Artemia a los 16 días, 2015.

Porcentaje de sobrevivencia general del ensayo. En la gráfica 6 se representa el porcentaje de sobrevivencia del ensayo general a los 16 días colocándose a eclosionar un total de 6000 huevos de Artemia estando distribuidos 1500 huevos por tratamiento obteniendo 3544 huevos eclosionados y una no eclosión de 2456 huevos donde para obtener el porcentaje de sobrevivencia general del ensayo se aplicaron las siguientes formulas. \% mortalidad: Numero de individuos / total de individuos * 100. Para obtener la sobrevivencia utilizamos la siguiente formula: \% sobrevivencia: $N$ - \% de mortalidad.

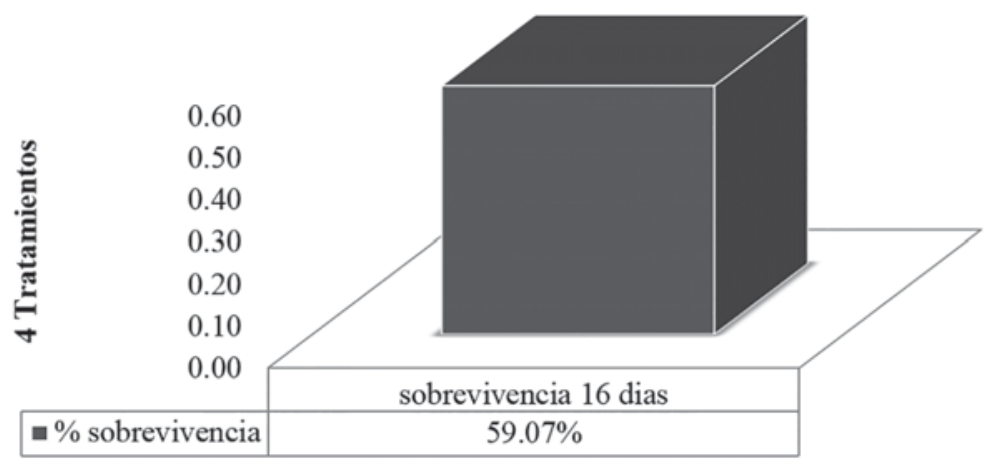

Figura 7. Porcentaje de sobrevivencia general del ensayo a los 16 días, 2015.
Recomendaciones para futuros cultivos de Artemia en laboratorio. Para evitar la evaporación del agua de los frascos y mantener el nivel de agua establecido y mantener control del $\mathrm{pH}$, es necesario mantener un recipiente alterno con agua desclorada y con un porcentaje de sal sin yodo e ir rellenando los tratamientos en estudios.

Para lograr una temperatura ambiente en el área donde se colocara el ensayo, es necesario apagar el aire acondicionado, abrir puertas y ventanas para que la sala tome la temperatura ambiente que haya en el día.

A la hora de alimentar al crustáceo es necesario triturar la pastilla hasta convertirla en polvo y dar la cantidad en gramos establecidos antes del ensayo.

A la hora de eliminar residuos de la alimentación del fondo de los frascos es necesario hacerlo con una jeringa para evitar golpear o estresar al crustáceo ya que podría provocarle la muerte.

\section{CONCLUSIONES}

Los tratamientos llevados a cabo fueron satisfactorios ya que se logró eclosionar el crustáceo A. franciscana logrando ser el tratamiento $\mathrm{D}$, el cual concluyo con un porcentaje de sobrevivencia arriba del 60\% lo que hace visible que el tratamiento D fue un poco más factibles para la eclosión del crustáceo.

El rendimiento de sobrevivencia de $A$. franciscana da pautas para seguir trabajando la eclosión de este crustáceo a nivel de laboratorio esto debido a que las condiciones ambientales que brinda la UNA en sus laboratorios son positivos para llevar a cabo trabajos investigativos.

Para que la eclosión del crustáceo A. franciscana se lleve a cabo, es necesario mantener una regulación del $\mathrm{pH}$ y que el local donde se ubique el ensayo permanezca la temperatura ambiente para no alterar el ciclo reproductivo de este.

\section{REFERENCIAS BIBLIOGRÁFICAS}

APROMAR. 2004. La acuicultura en el mundo. Cádiz. España. 9 p.

Castro, G. 2005. Importancia de los probióticos en la acuicultura, utilizando Artemia franciscana como bioencapsulante. México DF. Me. 5 p.

FAO (Organización de las Naciones Unidas para la Agricultura y la Alimentación). 1989. Descripción general de la especie Artemia franciscana. Managua, Nicaragua. 8 p.

Sorgeloos, N. 1986. La salinidad y su efecto en la reproducción del crustáceo Artemia sp. México DF. ME. 11 p.

Universidad de Colombia. 2002. Diseños de unidades experimentales. Colombia. 7 p. 